Ciência Florestal, Santa Maria, v. 27, n. 1, p. 263-275, jan.-mar., 2017

ISSN 1980-5098

\title{
TENDÊNCIAS DE CRESCIMENTO EM ALTURA DOMINANTE DE DOIS CLONES DE Eucalyptus saligna Smith IMPLANTADOS EM DISTINTAS CONDIÇÕES DE SOLO
}

\author{
DOMINANT HEIGHT GROWTH TRENDS OF TWO Eucalyptus saligna Smith CLONES \\ IMPLANTED ON DIFFERENT SOIL CONDITIONS
}

\author{
Lucas Zancan Pissinin ${ }^{1}$ Paulo Renato Schneider ${ }^{2}$
}

\begin{abstract}
RESUMO
Uma mesma espécie florestal pode, em distintas condições ambientais, apresentar variações na forma de seu crescimento e em consequência na sua produção final. A altura dominante $\left(\mathrm{h}_{100}\right)$ é uma das variáveis mais importantes para a prognose da produção florestal, visto que praticamente todas as demais relações funcionais de produção se baseiam na descrição do crescimento desta variável através das funções de índice de sítio. Este trabalho objetivou detectar as possíveis diferenças no crescimento em altura dominante $\left(\mathrm{h}_{100}\right)$ entre dois clones de uma mesma espécie (Eucalyptus saligna Smith) e entre diferentes classes de aptidão de solos, sobre os quais estão implantados estes materiais genéticos. Uma rede de parcelas permanentes com remedições anuais ou bianuais foi utilizada para ajustar diferentes modelos de altura dominante em função da idade. A partir da seleção do melhor modelo e do uso de análise de covariância, buscou-se definir a existência de diferentes tendências de crescimento em altura dominante. As análises de tendências de crescimento através da utilização da função de Backman demonstraram diferenças significativas entre os dois materiais genéticos e entre diferentes classes de aptidão de solos para o clone 2864. O clone 4039 não demonstrou tendências de crescimento suficientemente significativas que justificassem a separação em dois grupos de classes de solo. Como resultado final, tem-se que três grupos de crescimento deveriam ser formados para descrever o crescimento em altura dominante: Grupo I - Clone 2864 e classes de solo 1 e 2; Grupo II - Clone 2864 e classes de solo 3, 4 e 5 e Grupo III - Clone 4039 em todas as classes de solo. Palavras-chave: índice de sítio; manejo florestal; crescimento e produção florestal; solos florestais.
\end{abstract}

\begin{abstract}
A same species can, under different environmental conditions, show variations in its growth trend and consequently in its final production. The dominant height $\left(\mathrm{h}_{100}\right)$ is one of the most important variables for the forest growth and yield prediction, since we can see that a several number of yield functions are based on the dominant height description by site index equations. This paper had as objective to evaluate possible differences in the dominant height growth between two Eucalyptus saligna Smith clones and among different productivity soil classes, on which ones are implanted both Eucalyptus saligna clones. A set of permanent plots with annual or bi-annual measurements was used to fit different models of dominant height by age. Since the best model was defined and, utilizing the analysis of covariance, we tried to define the existence of different growth trends in dominant height. The growth trend analysis by using Backman's function showed significant differences between both clones and among the different soil classes for clone 2864. Clone 4039 did not show a growth trend sharply different which would enough to separate this clone in two soil based groups. As a final result, we have that three different growth groups should be created to appropriately describe the dominant height growth: Group I - Clone 2864 and soil classes 1 and 2; Group

1 Engenheiro Florestal, MSc., Coordenador de Operações Florestais - CMPC Celulose Riograndense, Rua São Geraldo, 1680, Bairro Ermo, CEP 92500-000, Guaíba (RS), Brasil. lucas.pissinin@cmpcrs.com.br

2 Engenheiro Florestal, Dr., Professor Titular do Departamento de Ciências Florestais, Centro de Ciências Rurais, Universidade Federal de Santa Maria, Av. Roraima, 1000, CEP 97105-900, Santa Maria (RS), Brasil. schneider. paulorenato@gmail.com
\end{abstract}

Recebido para publicação em 9/06/2013 e aceito em 15/04/2015

Ci. Fl., v. 27, n. 1, jan.-mar., 2017 
II - Clone 2864 and soil classes 3, 4 and 5 and Group III - Clone 4039 and all soil classes.

Keywords: site index; forest management; forest growth and yield; forest soils.

\section{INTRODUÇÃO}

As informações advindas de modelos apropriados de crescimento e produção são fundamentais para a determinação das idades de rotação, das densidades iniciais de plantio e do planejamento de intervenções na floresta (CLUTTER; FORTSON; PIENAAR, 1983). Modelos para predizer a produção futura têm assim, um papel muito importante como ferramenta de tomada de decisão, auxiliando a responder a perguntas tais como: Onde, quando, de que forma, e em que intensidade intervir na floresta? Dentro deste aspecto, a classificação dendrométrica dos sítios florestais, observando todas as suas sutis diferenças de comportamento, é uma das etapas de maior impacto na montagem de um sistema de prognose da produção florestal, isto porque a variável altura dominante $\left(\mathrm{h}_{100}\right)$ impactará sucessivamente em todas as demais funções de um modelo adequado de produção.

Existem diferentes métodos para a classificação da capacidade produtiva das áreas com florestas, podendo-se ainda agrupar todos eles em três diferentes categorias: avaliação pela vegetação indicadora, avaliação por fatores edafoclimáticos e avaliação pela relação altura dominante e idade (índices de sítio ou de local), sendo os dois primeiros considerados métodos indiretos, e o último como um método direto de classificação (GONÇALVES, 1988), embora Carvalho et al. (1999) classifiquem os mesmos como métodos extrínsecos e intrínsecos, respectivamente. O primeiro destes métodos, qual seja, a avaliação pela vegetação indicadora, apresenta-se ainda como o mais incipiente deles. Por outro lado, métodos de classificação por fatores edafoclimáticos, por índices de sítio e o estudo da correlação entre estes, têm sido amplamente estudados por diversos autores como Gonçalves (1988), Scolforo (1992), Carvalho et al. (1999), Tonini et al. (2002), Tonini (2003) e Tonini, Schneider e Finger (2006).

A densidade do solo, através da sua expressão na resistência à penetração das raízes, é fator de importância para o desenvolvimento das plantas. O crescimento de florestas de pinus no estado do Paraná, a exemplo, foi principalmente influenciado pela água disponível e pela resistência à penetração das raízes (BELLOTE; DEDECEK, 2006). O crescimento de Eucalyptus grandis (Hill) ex. Maiden até os três anos e meio de idade também foi afetado pela camada de impedimento em um solo da unidade de mapeamento Santa Maria no Rio Grande do Sul (FINGER et al., 1996). Observações semelhantes foram feitas por Ortiz et al. (2006) em um trabalho que relacionou os atributos físicos e químicos do solo com o potencial produtivo de florestas de eucalipto, expresso pelo seu índice de sítio. Nesse trabalho, os autores concluíram que os atributos físicos do solo, principalmente textura e estrutura, mostraram ter mais influência sobre a expressão do potencial da floresta através de seu índice de sítio.

As restrições químicas dos solos geralmente influenciam em taxas constantes o crescimento das florestas, o que faz com que povoamentos adubados e não adubados, a exemplo, tenham uma tendência de crescimento semelhante, embora sua produção final seja diferenciada. Neste sentido, Barros et al. (1984) detectaram que a tendência de crescimento de Eucalyptus saligna submetidos a tratamentos com e sem adubação de N:P:K foi similar, entretanto, a taxa de crescimento foi bastante superior naqueles tratamentos que receberam adubação. Restrições físicas, por outro lado, podem impactar de forma diferente o crescimento ao longo do ciclo da floresta. Solos rasos como exemplo, poderão oferecer suporte nutricional e hídrico adequados à floresta até determinada idade e, a partir daí, restringir o seu crescimento, devido ao sistema radicular das plantas terem explorado todo o potencial do solo quanto à sua capacidade de armazenamento e disponibilização de água. Segundo Campos e Leite (2009), o crescimento em altura dominante como exemplo, depende de condições usualmente ligadas a determinadas propriedades do solo, como a textura dos horizontes.

Tonini, Schneider e Finger (2006) detectaram diferentes tendências de crescimento em altura dominante de clones de Eucalyptus saligna Smith quando um mesmo clone era submetido a distintas condições de solo. Um dos clones avaliados demonstrou três tendências de crescimento, sendo que a tendência de crescimento sob cambissolos mostrou-se bastante diferenciada, gerando uma curva com maior achatamento, provavelmente devido às limitações deste tipo de solo quanto à sua profundidade e capacidade de armazenamento de água. Lourenço (1996), em estudo com Eucalyptus dunnii em uma 
topossequência, detectou diferenças no crescimento em diâmetro e altura aos sete anos de idade, tendo concluído que houve uma alta correlação entre a profundidade do horizonte A nos perfis avaliados com o crescimento em diâmetro.

Uma análise das observações dos autores citados permite inferir que as restrições de natureza química de determinados solos afetam significativamente a produção final e, as restrições de natureza física, além de afetarem a produção final de determinada espécie, podem afetar a forma como se desenvolverá esta espécie até atingir esta produção. A consideração das características dos solos quando das análises e modelagens do crescimento das florestas deve, portanto, ser reiterada, de forma que a utilização de técnicas específicas para este fim, incluindo-se variáveis qualitativas nas análises, pode ser uma ferramenta para aumentar a precisão e a confiabilidade dos estudos de crescimento e produção florestal. Considerando a importância do tema para o refinamento das técnicas de modelagem, este trabalho objetivou o estudo do crescimento em altura dominante de dois clones de Eucalyptus saligna Smith implantados em áreas com diferentes condições de solo, gerando assim subsídios para as etapas subsequentes da modelagem da produção destas florestas.

\section{MATERIAIS E MÉTODOS}

\section{Região de estudo, mapeamento de solos e materiais genéticos}

O Rio Grande do Sul está dividido em quatro regiões geomorfológicas: o planalto, a depressão periférica, o escudo sul-rio-grandense e a planície costeira (STRECK et al., 2008). A área de interesse do presente estudo encontra-se distribuída nas três últimas regiões, sendo que os municípios de São Gabriel, Viamão, Canguçu e Triunfo compreendem os limites oeste, leste, sul e norte da região de estudo, respectivamente.

Segundo a classificação de Köppen (1931), o clima do Estado do Rio Grande do Sul é dividido em duas variedades climáticas, $\mathrm{Cfa}$ e $\mathrm{Cfb}$. A variedade $\mathrm{Cfb}$ é restrita ao planalto superior (nordeste do estado) e à região do escudo sul-rio-grandense. As demais regiões do estado registram a variedade $\mathrm{Cfa}$ (MORENO, 1961).

As áreas nas quais estão implantados os povoamentos clonais de Eucalyptus saligna possuem um mapeamento de solo no nível de plantio (menor unidade de manejo), sendo que as informações de inventário para os clones descritos se encontram distribuídas em nove ordens de solos (argissolos, cambissolos, chernossolos, gleissolos, luvissolos, neossolos, nitossolos, planossolos e plintossolos). Estas nove ordens foram subcategorizadas em mais duas subordens, e outros dois níveis de classificação em relação à textura do solo e ao relevo. Posteriormente, os solos foram agrupados em cinco classes de aptidão (apta superior, apta inferior, marginal superior, marginal inferior e inferior ou inapta) para o cultivo de Eucalyptus, partindo-se do conceito desenvolvido no trabalho realizado por Costa et al. (2009).

A Tabela 1 resume o número de diferentes tipos de solos mapeados por classe de aptidão e ordem (conforme classificação de primeiro nível). As variáveis IQS e CAD refletem o Índice de Qualidade do Solo e a Capacidade de Água Disponível, respectivamente. No mapeamento de solos da empresa Celulose Riograndense Ltda., o IQS (Índice de qualidade do solo) foi determinado com sendo o somatório ponderado das seguintes características dos solos: armazenar e suprir água, promover o crescimento das raízes, armazenar, ciclar e suprir nutrientes e promover atividade biológica, sendo cada uma destas características associadas às características químicas, físicas e biológicas dos solos. Detalhes metodológicos desta classificação são encontrados em Freitas et al. (2012), Mendes et al. (2008) e Melo Filho et al. (2007). A variável CAD (Capacidade de água disponível) por sua vez, foi determinada neste mesmo mapeamento, conforme metodologia de Libardi (2010).

Os plantios clonais estabelecidos na área de estudo, pertencentes à empresa Celulose Riograndense Ltda., compreendem hoje uma área significativa de cerca de $60 \%$ do total de plantios da companhia, sendo que os clones de Eucalyptus saligna destacam-se pela sua produtividade e grande plasticidade a diferentes sítios. Dois materiais genéticos de Eucalyptus saligna, denominados clone 2864 e clone 4039, são hoje os materiais mais plantados. Devido à sua grande área plantada e consequente importância para os planos de suprimento de matéria-prima para a fabricação de celulose, estes dois clones foram selecionados para a 
realização deste estudo.

\section{Parcelas permanentes e metodologia de análise das informações}

Este trabalho utilizou-se de um banco de dados com medições de parcelas permanentes dos materiais genéticos descritos no item anterior. Estas parcelas foram remedidas anual ou bianualmente, a fim de proporcionar dados sequenciais dos parâmetros de crescimento dos povoamentos. Todas as parcelas foram instaladas em formato circular, de raio $11,29 \mathrm{~m}$. Dentro de cada parcela foram tomados todos os diâmetros a altura do peito (DAP) a 1,30 m do nível do solo e as alturas das duas fileiras centrais, bem como as alturas das árvores dominantes, segundo conceito de Assmann, em que se consideram dominantes as 100 árvores por hectare com maior DAP.

As informações geradas pelas remedições de cada parcela foram basicamente o número de árvores vivas, mortas e falhas; o diâmetro (DAP) médio, mínimo, máximo e quadrático; altura média, mínima, máxima e altura dominante; os coeficientes de variação do diâmetro (DAP) e altura; a área basal, e os volumes comerciais com e sem casca.

Um total de 6800 parcelas foi utilizado neste estudo. Destas, 2253 parcelas possuíam de duas até seis remedições, ou seja, foram remedidas pelo menos em uma ocasião, totalizando 5013 medições. As demais 4547 parcelas foram medidas em apenas uma ocasião até o momento. As parcelas com pelo menos uma remedição estavam distribuídas inicialmente na seguinte magnitude para cada classe de aptidão, conforme mapeamento de solos (de 1 a 5; da classe superior até a inapta ao cultivo): 1651 parcelas na classe 1; 2100 na classe 2; 903 na classe 3; 161 na classe 4 e 198 na classe 5. A Figura 1 demonstra a distribuição de todas as medições das parcelas com pelo menos uma remedição nas diferentes classes de idade e de aptidão de solo para cada material genético.

Os modelos, conforme a Tabela 2, foram ajustados para todo o conjunto de dados, gerando-se cinco diferentes estatísticas de avaliação. O programa estatístico "Statistica 9.1", licenciado para Celulose Riograndense Ltda., foi utilizado para, através do método "General Linear Models" - "Multiple Regression" e do método "Nonlinear-Estimation", ajustar os modelos lineares e não lineares, respectivamente. Para cada modelo foram estabelecidas as avaliações de coeficiente de determinação ajustado $\left(\mathrm{R}_{\text {aj }}{ }\right)$, distribuição gráfica dos resíduos, estabilidade das curvas geradas, erro padrão residual percentual (Syx\%) e índice de Furnival (IF\%).

A estatística coeficiente de determinação $\left(\mathrm{R}^{2}\right)$, para as equações lineares, foi calculada pela razão entre a soma dos quadrados da regressão e a soma dos quadrados total:

$$
\mathrm{R}^{2}=\frac{\mathrm{SQ}_{\text {regressão }}}{\mathrm{SQ}_{\text {total }}}
$$

Para as equações não lineares, o coeficiente de determinação foi calculado por:

$$
\mathrm{R}^{2}=1-\left(\frac{\mathrm{SQ}_{\text {resíduos }}}{S \mathrm{Q}_{\text {total corrigido }}}\right)
$$

O coeficiente de determinação foi corrigido para o número de variáveis independentes das equações, determinando-se o $\mathrm{R}^{2}$ ajustado pela fórmula:

$$
\mathrm{R}^{2} \text { aj }=\mathrm{R}^{2}-\left(\frac{\mathrm{K}-1}{\mathrm{~N}-\mathrm{K}}\right) \cdot\left(1-\mathrm{R}^{2}\right)
$$

Em que: $\mathrm{K}=$ número de variáveis independentes da equação; $\mathrm{N}=$ número de observações; $\mathrm{R}^{2}=$ coeficientes de determinação; $\mathrm{SQ}=$ Soma de quadrados.

A avaliação gráfica dos resíduos levou em consideração a tendenciosidade ou não de cada modelo e não necessariamente a dispersão dos resíduos, visto que uma função ajustada para altura dominante representa apenas a regressão média ou curva-guia sobre a qual, posteriormente, serão construídas as curvas de índice de sítio, que, por sua vez, deverão cobrir essa variação de altura para cada idade.

Para a verificação da estabilidade das curvas de sítio pelos modelos ajustados, selecionaram- 


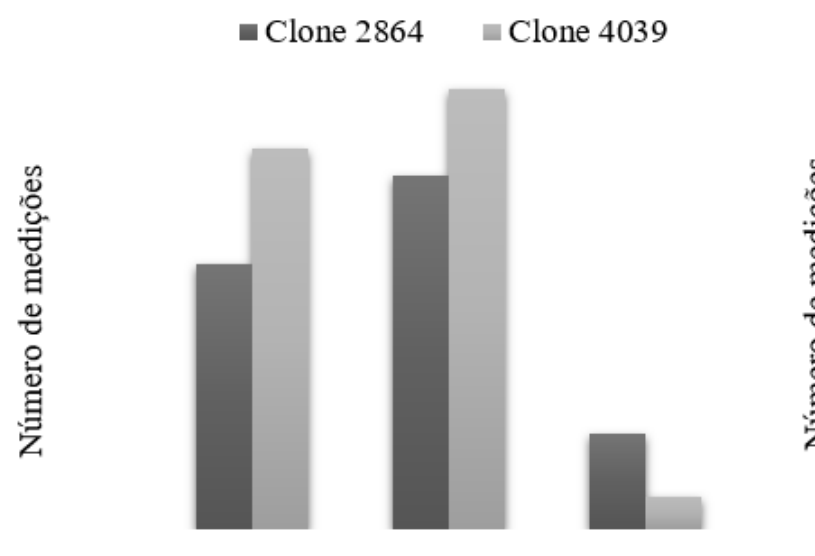

Classe de idade da medição (anos)

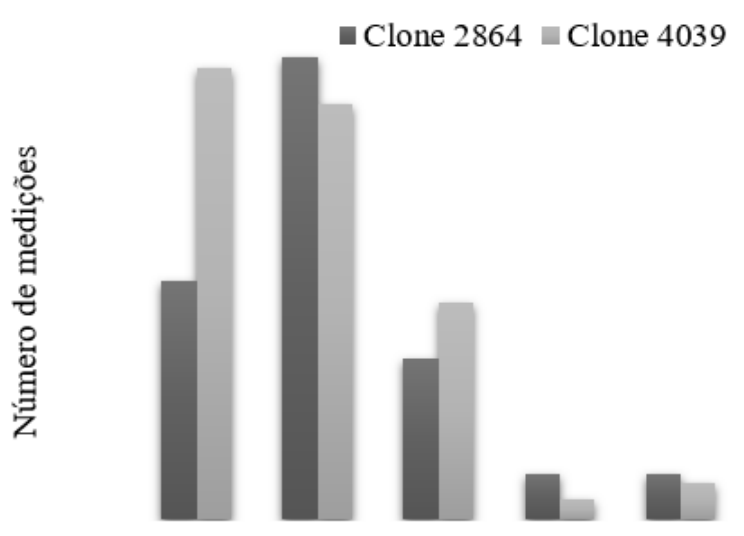

Classe de aptidão de solos

FIGURA 1: Distribuição das medições de parcelas permanentes nas diferentes classes de idade e classes de solo, para cada clone de Eucalyptus saligna no estado do Rio Grande do Sul.

FIGURE 1: Measure distribution in the different age classes and soil classes, for each Eucalyptus saligna clone evaluated in Rio Grande do Sul state.

TABELA 1: Distribuição dos solos por classe de produtividade (aptidão) e valores médios de índice de qualidade do solo (IQS) e capacidade de água disponível (CAD) nas áreas florestais da empresa Celulose Riograndense Ltda.

TABLE 1: Soil distribution by productivity classes and average values of soil quality index (IQS) and available water capacity (CAD) at Celulose Riograndense forest areas.

\begin{tabular}{ccccc}
\hline $\begin{array}{c}\text { Classe de } \\
\text { aptidão }\end{array}$ & Ordem de Solos & $\begin{array}{c}\text { Número de solos } \\
\text { descritos }\end{array}$ & IQS & CAD \\
\hline \multirow{2}{*}{1} & Argissolo & 2 & 0,80 & 428 \\
& Nitossolo & 1 & 0,90 & 348 \\
\hline \multirow{2}{*}{2} & Argissolo & 7 & 0,71 & 300 \\
& Cambissolo & 2 & 0,73 & 248 \\
& Chernossolo & 4 & 0,70 & 383 \\
& Luvissolo & 3 & 0,65 & 238 \\
& Neossolo & 2 & 0,70 & 143 \\
& Nitossolo & 1 & 0,87 & 348 \\
\hline \multirow{3}{*}{3} & Argissolo & 8 & 0,62 & 244 \\
& Cambissolo & 6 & 0,63 & 248 \\
& Luvissolo & 1 & 0,70 & 241 \\
& Neossolo & 1 & 0,65 & 40 \\
\hline & Argissolo & 2 & 0,53 & 250 \\
& Cambissolo & 2 & 0,55 & 233 \\
& Neossolo & 2 & 0,50 & 89 \\
& Planossolo & 1 & 0,50 & 225 \\
& Plintossolo & 3 & 0,50 & 350 \\
\hline \multirow{2}{*}{5} & Gleissolo & 3 & 0,40 & 184 \\
& Planossolo & 2 & 0,45 & 214 \\
\hline
\end{tabular}

Em que: IQS = Índice de qualidade do solo; $\mathrm{CAD}$ = Capacidade de água disponível. Fonte: Mapeamento de solos da empresa Celulose Riograndense Ltda. 
TABELA 2: Modelos avaliados para descrever o crescimento em altura dominante para os dois clones de Eucalyptus saligna.

TABLE 2: Evaluated models to describe the dominant height growth of the two Eucalyptus saligna clones.

\begin{tabular}{lccc}
\hline \multicolumn{1}{c}{ Modelo } & Método & Forma de Ajuste & Bibliografia \\
\hline $\begin{array}{l}\text { Schumacher 3 } \\
\text { parâmetros }\end{array}$ & Curva-Guia & $\mathrm{Lnh}_{100}=\mathrm{b}_{0}+\mathrm{b}_{1} \times \mathrm{t}^{\mathrm{b}_{2}}$ & Campos e Leite (2006) \\
Backman & Curva-Guia & $\mathrm{Lnh}_{100}=\mathrm{b}_{0}+\mathrm{b}_{1} \times \mathrm{Ln}(\mathrm{t})+\mathrm{b}_{2} \times \mathrm{Ln}^{2}(\mathrm{t})$ & Tonini (2003) \\
Prodan & Curva-Guia & $\mathrm{h}_{100}=\mathrm{t}^{2} /\left(\mathrm{b}_{0}+\mathrm{b}_{1} \times \mathrm{t}+\mathrm{b}_{2} \times \mathrm{t}^{2}\right)$ & Tonini (2003) \\
Chapman-Richards & Curva-Guia & $\mathrm{h}_{100}=\mathrm{b}_{0} \times\left(1-\exp \left(-\mathrm{b}_{1} \times \mathrm{t}\right)\right)^{\left(\frac{1}{1-\mathrm{b}_{2}}\right)}$ & Clutter (1983) \\
$\begin{array}{l}\text { Schumacher 3 } \\
\text { parâmetros }\end{array}$ & $\begin{array}{l}\text { Diferença } \\
\text { Algébrica }\end{array}$ & $\left.\mathrm{Lnh}_{100 \mathrm{f}-\mathrm{Lnh}_{100 \mathrm{i}}=\mathrm{b}_{0} \times\left(\left(\frac{1}{\mathrm{t}_{\mathrm{f}}}\right)^{\mathrm{b}_{2}}-\left(\frac{1}{\mathrm{t}_{\mathrm{i}}}\right)^{\mathrm{b}_{2}}\right.}\right)$ & Campos e Leite (2006) \\
& $\begin{array}{c}\text { Diferença } \\
\text { Chapman-Richards }\end{array}$ & $\mathrm{h}_{100 \mathrm{f}}=\mathrm{h}_{100 \mathrm{i}} \times \frac{\left(\left(1-\exp \left(-\mathrm{b}_{1} \times \mathrm{t}_{\mathrm{f}}\right)\right)^{\left(\frac{1}{1-\mathrm{b}_{2}}\right)}\right.}{\left(\left(1-\exp \left(-\mathrm{b}_{1} \times \mathrm{t}_{\mathrm{i}}\right)\right)^{\left(\frac{1}{1-\mathrm{b}_{2}}\right)}\right.}$ & Clutter (1983)
\end{tabular}

Em que: $\mathrm{h}_{100}=$ Altura dominante (m), segundo conceito de Assmann; $\mathrm{t}=$ Idade (anos); $\mathrm{Ln}=$ logaritmo de base natural; $\exp =$ Função exponencial inversa do Ln; $h_{100 f}=$ Altura dominante na idade final; $h_{100 i}=$ Altura dominante na idade inicial; $\mathrm{t}_{\mathrm{f}}=$ Idade final; $\mathrm{t}_{\mathrm{i}}=$ Idade inicial.

se aleatoriamente, do banco de dados, 72 parcelas com três ou mais medições. A partir destas parcelas, para cada remedição e com cada modelo, estimaram-se os respectivos índices de sítio. O percentual de estabilidade foi obtido pela razão entre somatório das parcelas que durante todas as remedições mudaram de sítio no máximo uma vez e o número total de parcelas avaliado.

O erro padrão residual percentual foi obtido pela seguinte expressão:

$$
\operatorname{Syx} \%=\left(\frac{\sqrt{\left(\mathrm{QM}_{\mathrm{res}}\right)}}{\overline{\overline{\mathrm{Y}} \text { Var. Dep. }}}\right) \cdot 100
$$

Em que: Syx\% = Erro padrão residual percentual; $\mathrm{QM}_{\mathrm{res}}=$ Quadrado médio dos resíduos; $\overline{\mathrm{Y}}$ Var. Dep. $=$ Média aritmética da variável dependente.

O índice de Furnival foi calculado através da metodologia proposta por Silva e Bailey (1991), na qual se propôs um fator de correção sobre o índice originalmente proposto por Furnival (1961).

Originalmente, o índice era calculado por:

$$
\mathrm{IF}=\operatorname{antilog}\left(\frac{\sum_{\mathrm{i}=0}^{\mathrm{n}}\left(\log \left(\mathrm{B}^{\prime}\right)\right)}{\mathrm{n}}\right)^{-1} \cdot \operatorname{Syx}
$$

Com a modificação proposta, o índice passa a ser calculado pela expressão:

$$
\mathrm{IF}=\operatorname{antilog}\left(\frac{\sum_{\mathrm{i}=0}^{\mathrm{n}}\left(\log \left(\mathrm{B}^{\prime}\right)\right)}{\mathrm{n}}\right)^{-1} \cdot \operatorname{Syx} \cdot\left(\exp \left(\frac{\mathrm{n}-\mathrm{p}}{2 * \mathrm{n}}\right)\right) \quad \mathrm{IF} \%=\left(\frac{\mathrm{IF}}{\overline{\mathrm{Y}}}\right) \cdot 100
$$

Em que: IF = Índice de Furnival; IF\% = Índice de Furnival Percentual; Syx = Erro padrão residual; $\mathrm{n}=$ número de observações; $\log \left(\mathrm{B}^{\prime}\right)=\operatorname{logaritmo~da~variável~dependente;~} \mathrm{p}=$ número de parâmetros da equação. 
TABELA 3: Estatísticas avaliadas em cada modelo testado para clones de Eucalyptus saligna.

TABLE 3: Evaluated statistics used to test each model for Eucalyptus saligna clones.

\begin{tabular}{lcccccc}
\hline \multicolumn{1}{c}{ Função } & Método & $\mathrm{R}^{2}{ }_{\text {aj }}$ & Resíduos & Est. \% & Syx\% & IF \% \\
\hline Schumacher 3 parâmetros & Curva-Guia & 0,82 & sem tend. & 47 & 6,4 & 0,1 \\
Backman & Curva-Guia & 0,82 & sem tend. & 57 & 6,0 & 0,1 \\
Prodan & Curva-Guia & 0,89 & sem tend. & 53 & 13,2 & 6,6 \\
Chapman-Richards & Curva-Guia & 0,89 & sem tend. & 49 & 13,3 & 6,6 \\
Schumacher 3 parâmetros & Eq. das diferenças & 0,72 & sem tend. & 58 & 22,2 & 1,0 \\
Chapman-Richards & Eq. das diferenças & 0,71 & Moderada & 57 & 9,3 & 4,6 \\
\hline
\end{tabular}

Em que: $\mathrm{R}^{2}$ aj.= Coeficiente de determinação ajustado; Syx $\%=$ Erro padrão residual percentual; IF \% = Índice de Furnival (\%); Est $(\%)=$ percentual de estabilidade obtido pelas curvas.

A justificativa para essa proposição é a de que o índice como foi originalmente concebido não levava em consideração o número de parâmetros das funções, fazendo com que aquelas que tivessem o menor número de parâmetros fossem favorecidas por tal avaliação (SILVA; BAILEY, 1991). Pela utilização do índice originalmente descrito, o mesmo deveria ser calculado para modelos com transformação da variável dependente e comparados com o Syx \% dos modelos sem transformação. Devido a isso, na proposição original, o IF\% calculado para modelos sem transformação, é igual ao seu erro padrão residual em porcentagem. Sob esta nova ótica, o IF\% passa a ser uma nova estatística de avaliação, não devendo mais ser comparado com o Syx $\%$, mas sim com o próprio $\mathrm{IF} \%$, agora calculado para todos os modelos, com e sem transformação da variável dependente.

A função selecionada foi utilizada para, através da análise "General Linear Models" - "Homogeneity of slopes model" do software Statistica 9.1, licenciado para Celulose Riograndense Ltda., indicar diferenças estatísticas significativas de interação entre os fatores material genético e solo e o crescimento em altura dominante. Primeiramente, testou-se a existência de diferentes comportamentos no crescimento em altura dominante entre os dois clones. Caso tal diferença fosse significativa, ou seja, sugerisse a formação de dois grupos de curvas separando-se os materiais genéticos, a análise do fator solo seria realizada separadamente para cada clone. Este procedimento estatístico de análise das tendências de crescimento também é mais comumente conhecido como análise de covariância e neste estudo, para efeitos de terminologia, os dois termos utilizados significarão o mesmo tipo de procedimento estatístico.

\section{RESULTADOS E DISCUSSÃO}

\section{Seleção dos modelos para descrever o crescimento em altura dominante}

Cada modelo, para descrever o crescimento em altura dominante, foi ajustado com todo o conjunto de dados, gerando as estatísticas $\mathrm{R}_{\text {aj }}$, tendências nos resíduos, estabilidade das curvas ajustadas, erro padrão residual e índice de Furnival. Os resultados da Tabela 3 demonstram que praticamente todos os modelos apresentaram adequados valores de $\mathrm{R}^{2}{ }_{\mathrm{aj}}$. Visto que, nos ajustes dos modelos de altura dominante em função da idade, a curva média ajustada representa apenas o índice de sítio médio e toda a variação ao redor dessa curva é explicada pelas demais curvas de índice de sítio, inferiores e superiores à curva média, valores de $\mathrm{R}^{2}{ }_{\text {aj }}$ acima de 0,8 são considerados com bons ajustes para estes tipos de modelos.

A distribuição dos resíduos foi semelhante e sem tendências significativas para todos os modelos testados, a exceção do modelo de Chapman-Richards, ajustado pelo método da equação das diferenças. A estabilidade das curvas foi significativamente maior nas equações de Backman, Schumacher e ChapmanRichards, a primeira ajustada pelo método da curva-guia, e as duas últimas pelo método da equação das diferenças. O erro padrão residual percentual e o índice de Furnival também foram inferiores nas funções de Schumacher e Backman.

Para a seleção do melhor modelo, montou-se uma matriz de seleção (Tabela 4), na qual cada modelo 
TABELA 4: Hierarquização dos modelos ajustados para clones de Eucalyptus saligna para cada estatística calculada.

TABLE 4: Classification of the fitted models for Eucalyptus saligna clones to each calculated statistic.

\begin{tabular}{lcccccc}
\hline \multicolumn{1}{c}{ Matriz de seleção } & $\mathrm{R}^{2}{ }_{\text {aj }}$ & Resíduos & Estabilidade & Syx\% & IF \% & Totais \\
\hline Schumacher 3 parâmetros & 2 & 1 & 5 & 2 & 1 & 11 \\
Backman & 2 & 1 & 2 & 1 & 1 & 7 \\
Prodan & 1 & 1 & 3 & 4 & 4 & 13 \\
Chapman-Richards & 1 & 1 & 4 & 5 & 4 & 15 \\
Schumacher 3 parâmetros & 3 & 1 & 1 & 6 & 2 & 13 \\
Chapman-Richards & 4 & 2 & 2 & 3 & 3 & 14 \\
Em que: $\mathrm{R}^{2}$ aj.= Coeficiente de determinação ajustado; Syx \% = Erro padräo residual percentual; $1 \mathrm{~F} \%$ = Indice de
\end{tabular}

Furnival (\%); Est $(\%)=$ percentual de estabilidade obtido pelas curvas.

foi hierarquizado de 1 a n para cada estatística avaliada. O somatório das pontuações em cada estatística gerou um valor para o modelo, sendo escolhido o de menor pontuação como o que melhor descreveu o crescimento em altura dominante para todo o conjunto de dados. Pela análise de todas as estatísticas e a ponderação das mesmas através da matriz de seleção dos modelos, conclui-se que, para todo o conjunto de dados, o modelo de Backman (modelo linear) foi o que se mostrou mais adequado para representar o crescimento em altura dominante para os clones de Eucalyptus saligna. Dentre os modelos não lineares, o modelo de Schumacher com 3 parâmetros ajustados pelo método da curva-guia apresentou-se como o mais adequado.

O mesmo modelo de Backman foi utilizado com sucesso por Tonini (2003), que descreveu as tendências de crescimento em altura dominante para clones do gênero Eucalyptus. No estudo, o autor também utilizou esta função para realizar testes de nível e inclinação nas curvas, definindo padrões de crescimento para diferentes materiais genéticos e outros tratamentos como preparo de solo, adubação, tipo de solo, posição no relevo, exposição e inclinação. Selle (2009) também utilizou esta mesma função para descrever o crescimento em altura dominante em distintos sítios implantados com Hovenia dulcis Thunberg na região central do estado do Rio Grande do Sul, demonstrando que a função de Backman é bastante flexível para descrever diferentes tendências de crescimento.

$\mathrm{Na}$ maioria dos casos, apenas um segmento de toda a vida da floresta é avaliado nos estudos de crescimento e produção com florestas plantadas no Brasil, nos quais geralmente os dados coletados em inventários contínuos nesse tipo de floresta não englobam idades inferiores nem superiores aos pontos de inflexão de crescimento. Isto pode levar este segmento a ser representado por uma função quadrática, na qual a função de Backman acaba por descrever de maneira satisfatória essas tendências. Uma função com propriedades biológicas, como por exemplo, a função de Chapman-Richards, na qual a curva parte do valor "zero" da variável dependente, quando a variável independente também for "zero", e que estabelece uma assíntota e pode, muitas vezes, "forçar" um ajuste que na realidade não está presente nos dados.

\section{Análises das tendências de crescimento}

A partir da definição da função de Backman como sendo a que melhor descreve todo o conjunto de dados de altura dominante e idade, procederam-se as análises do comportamento das curvas para cada fator analisado: material genético (clones) e classes de aptidão de solo. A análise de covariância do fator material genético indicou haver diferenças significativas entre os clones estudados. Na Tabela 5 pode ser observada a significância estatística para este fator a um nível de probabilidade de erro de $1 \%$.

Ambas as variáveis independentes do modelo, $\operatorname{Ln}(\mathrm{t})$ e $\operatorname{Ln}^{2}(\mathrm{t})$ sofrem influência significativa do fator material genético, representada pelos valores de 0,00010 e 0,00432 para a estatística $p$ (significância), indicando diferenças na inclinação das curvas de crescimento. A diferença em nível das curvas também é indicada pela significância do fator de análise ( $\mathrm{MG}$ - material genético). Em termos práticos, este 
TABELA 5: Análise de covariância para o fator material genético.

TABLE 5: Analysis of covariance for the genetic material factor (clone).

\begin{tabular}{lccccc}
\hline \multicolumn{1}{c}{ Efeito } & SQ & GL & QM & F & p \\
\hline Intercepto & 13,53025 & 1 & 13,53025 & 529,71177 & 0,00000 \\
MG & 0,60825 & 1 & 0,60825 & 23,81313 & 0,00000 \\
$\mathrm{Ln}(\mathrm{t})$ & 0,49718 & 1 & 0,49718 & 19,46480 & 0,00001 \\
$\mathrm{Ln}(\mathrm{t})$ & 0,11603 & 1 & 0,11603 & 4,54256 & 0,03309 \\
$\mathrm{MG} * \operatorname{Ln}(\mathrm{t})$ & 0,38910 & 1 & 0,38910 & 15,23343 & 0,00010 \\
$\mathrm{MG} * \operatorname{Ln}^{2}(\mathrm{t})$ & 0,20810 & 1 & 0,20810 & 8,14713 & 0,00432 \\
$\operatorname{Ln}(\mathrm{t}) * \operatorname{Ln}^{2}(\mathrm{t})$ & 0,36354 & 1 & 0,36354 & 14,23277 & 0,00016 \\
$\mathrm{MG} * \operatorname{Ln}(\mathrm{t}) * \operatorname{Ln}^{2}(\mathrm{t})$ & 0,09914 & 1 & 0,09914 & 3,88150 & 0,04885 \\
\hline Erro & 244,03461 & 9554 & 0,02554 & &
\end{tabular}

Em que: $\mathrm{SQ}=$ Soma de quadrados; $\mathrm{GL}=$ Graus de liberdade; $\mathrm{QM}=$ Quadrado médio; $\mathrm{F}=$ Estatística F; $\mathrm{p}=$ Valor de probabilidade "p"; $M G$ = Material genético (clone); $\mathrm{t}=$ Idade.

resultado indica que, apesar destes materiais genéticos serem ambos de clones da mesma espécie, possuem comportamentos distintos de crescimento, sugerindo assim a formação de pelo menos dois grupos de curvas de índice de sítio. Esta diferença entre clones também pode ser claramente observada na Figura 2. Nela, o modelo de Backman foi previamente ajustado na sua forma linearizada, separadamente para cada clone, com o intuito de demonstrar graficamente o resultado estatístico obtido.

O clone 4039 apresenta, a partir de dois anos e meio, um crescimento mais acentuado em altura dominante, porém, próximos aos sete anos, o clone 2864 tem em média sua altura maior que o primeiro. Esta característica é provavelmente inerente ao genótipo de cada material genético e tem forte influência sobre as curvas de crescimentos de outras variáveis dendrométricas derivadas da altura dominante, como diâmetro, área basal e volume.

Trugilho et al. (2001) detectaram diferenças de crescimento em altura média, diâmetro e volume para diferentes clones de mesma espécie. Os autores estudaram sete diferentes materiais genéticos de

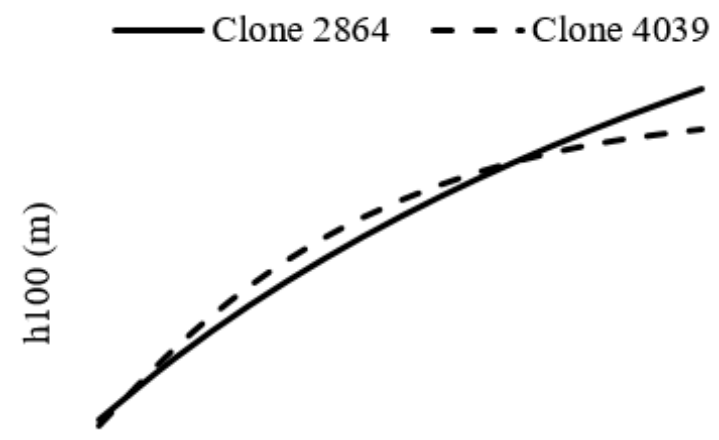

Idade (anos)

FIGURA 2: Curvas ajustadas pelo modelo de Backman para os clones 2864 e 4039 de Eucalyptus saligna após detecção de diferença significativa pelo fator material genético.

FIGURE 2: Fitted curves by Backman's model for the Eucalyptus saligna clones, 2864 and 4039, after detecting significant difference for the genetic material factor (clone). 
Eucalyptus grandis e três materiais de Eucalyptus saligna, concluindo que existiam diferenças significativas entre clones de uma mesma espécie, principalmente nos clones de Eucalyptus saligna. Como os plantios avaliados neste estudo encontram-se regularmente distribuídos em diferentes sítios, pode-se concluir que as diferenças detectadas são consistentes e advindas das características genotípicas de cada clone e, portanto, dever-se-iam manter dois grupos de crescimento em altura dominante (um para cada clone) para as análises de tendência de crescimento nas diferentes classes de aptidão de solos.

A partir desta detecção de diferença significativa entre clones, analisou-se, para cada um deles, a existência de diferenças significativas entre cada classe de aptidão de solo. A Tabela 6 resume os resultados encontrados em cada análise cruzada, mostrando o valor de probabilidade "p" associada à interação do fator com a variável independente $\operatorname{Ln}(\mathrm{t})$ do modelo de Backman: . Pela observação dos resultados podese concluir a existência de diferenças significativas entre algumas classes de aptidão de solos, embora não se possa identificar claramente algum tipo de agrupamento entre classes semelhantes. Quando ocorre a separação do banco de dados nestas classes, o número de observações de cada uma delas diminui e, portanto, a significância estatística pode ser influenciada pelo número de observações em cada classe. Desta forma, esta primeira análise serviu apenas para indicar possíveis agrupamentos de classes, já que não houve diferenças entre todas elas, e assim não se justificaria a separação nas cinco classes.

Para o clone 2864, observa-se que a classe 3 diferenciou-se das demais, exceto da classe 5. Já para o clone 4039, a classe 5 mostrou-se significativamente diferente das demais, exceto da classe 4. Para a tomada de decisão a respeito de que agrupamentos poderiam resultar significativamente distintos e lógicos, optouse por sugerir um agrupamento de classes único para os dois clones. Assim, a classe 3, que se mostrou diferente para o clone 2864 e as classes 4 e 5 (diferentes para o clone 4039) e que não diferiram entre si, formaram novos grupos, complementando-se com as classes 1 e 2. Esta sugestão de agrupamento é a mais lógica quanto às características de cada solo, conforme descritas na Tabela 1 . Desta forma, repetiram-se as análises cruzadas agora com os grupos de solos "1-2"; "3" e "4-5", para ambos os clones. Os resultados podem ser encontrados na Tabela 7.

Nesta nova análise, as diferenças estatísticas foram confirmadas entre classes. Assim, pode-se facilmente concluir de forma lógica que, para o clone 2864, a nova classe 1-2 diferiu das demais, sugerindo que sejam construídos dois sistemas de curvas distintos: um para os grupos de solos 1-2 (Grupo I) e outro para os grupos 3-4-5 (Grupo II). Da mesma forma, para o clone 4039, há claramente a sugestão de separação

TABELA 6: Significância representada pelo valor de probabilidade "p" entre as classes de aptidão dos solos pela análise de covariância para os clones 2864 e 4039 de Eucalyptus saligna.

TABLE 6: Significance demonstrated by the probability "p" value among different soil classes, by using the analysis of covariance for the clones 2864 and 4039 of Eucalyptus saligna.

\begin{tabular}{ccccccc}
\hline & Classe x Classe & 1 & 2 & 3 & 4 & 5 \\
\cline { 2 - 7 } Clone 2864 & 1 & -- & 0.05425 & 0.00060 & 0.36648 & 0.09677 \\
& 2 & 0.05425 & -- & 0.00000 & 0.81407 & 0.00321 \\
& 3 & 0.00060 & 0.00000 & -- & 0.00946 & 0.33571 \\
& 4 & 0.36648 & 0.81407 & 0.00946 & -- & 0.09646 \\
& 5 & 0.09677 & 0.00321 & 0.33571 & 0.09646 & -- \\
\hline \multirow{4}{*}{ Clone 4039 } & Classe x Classe & 1 & 2 & 3 & 4 & 5 \\
\cline { 2 - 7 } & 1 & -- & 0.00514 & 0.43066 & 0.90656 & 0.00002 \\
& 2 & 0.00514 & -- & 0.08557 & 0.50014 & 0.00565 \\
& 3 & 0.43066 & 0.08557 & -- & 0.23184 & 0.00042 \\
& 4 & 0.90656 & 0.50014 & 0.23184 & -- & 0.25098 \\
\hline
\end{tabular}

Em que:Valores de probabilidade $\mathrm{p}<0,01$ em negrito indicam diferenças significativas de inclinação ou nível pela análise de covariância entre as diferentes classes de aptidão de solos sob um nível de probabilidade de erro de 1\%. 
em dois grupos, um formado pelas classes 1-2-3 (Grupo III) e outro pelas classes 4-5 (Grupo IV).

Pelos resultados expressos na Figura 3, observa-se que para o clone 2864 é recomendado a manutenção dos grupos I e II de forma separada, pois as diferenças estatísticas foram comprovadas pelo gráfico da curva média ajustada e pelas diferenças crescentes de altura a partir dos cinco/seis anos de idade, o que gerou um comportamento bastante distinto de crescimento. As classes de solos 1 e 2, que compreendem basicamente Argissolos Vermelhos, Argissolos Vermelho-Amarelos, Nitossolo Vermelho, alguns Cambissolos, Chernossolos e Luvissolos, com valores médios de IQS de 0,76 e CAD 304 mostraramse superiores em crescimento a partir de cinco anos de idade. Por outro lado, e de forma lógica, as classes 3, 4 e 5 com valores médios de IQS e de CAD de 0,55 e 211, respectivamente, apresentaram menor crescimento em altura dominante. O maior crescimento em altura dominante do grupo formado por solos de melhor qualidade (classes 1 e 2) deve estar indicando a maior capacidade desses solos em sustentar o crescimento, dando maior suporte hídrico e nutricional à floresta à medida que as árvores crescem e demandam mais do sítio.

Para o clone 4039, as diferenças estatísticas não se mostraram claras quando os grupos obtiveram seus ajustes em separado. Observa-se, pela Figura 3, que as diferenças entre os grupos são pequenas durante todo o período de desenvolvimento analisado. Outra observação importante é a de que o grupo III (clone 4039 + solos 1-2-3) chega aos oito anos com um crescimento em altura menor do que o grupo IV (clone 4039 + solos 4-5) que apresenta solos com maior limitação por excesso de água e má-drenagem. Este resultado também pode indicar que o volume de dados para ajustar cada grupo separadamente não foi suficientemente adequado, sugerindo-se então que para o clone 4039 não se faça a separação pelo fator solo. Devido a isso, optou-se por manter o clone 4039 como um único grupo, formado por todas as classes de solo.

Assim como o demonstrado para o clone 2864, os atributos físicos do solo podem influenciar significativamente o desenvolvimento de florestas de Eucalyptus. As características como camadas de impedimento, diferentes graus de compactação, textura e estrutura do solo foram detectadas como sendo influenciadoras no desenvolvimento de plantios do gênero no Rio Grande do Sul e no estado de São Paulo (FINGER et al., 1996; ORTIZ et al., 2006). Diferentes tipos de solos podem influenciar diferenças no crescimento em altura e diâmetro, segundo Lourenço (1996). O autor encontrou que a espécie Eucalyptus dunnii cresceu de forma diferenciada em latossolos e cambissolos, sendo que nos primeiros, a floresta atingiu maiores diâmetros e alturas aos sete anos de idade.

Neste estudo, apenas um dos clones avaliados apresentou diferenças claras que justificassem a separação em distintos grupos de crescimento em função do solo, entretanto, mesmo o clone 4039 demonstrou estatisticamente algumas diferenças que podem ser consideradas para estudos posteriores em que se disponha de uma maior quantidade de medições em idades mais avançadas (maiores de 10 anos).
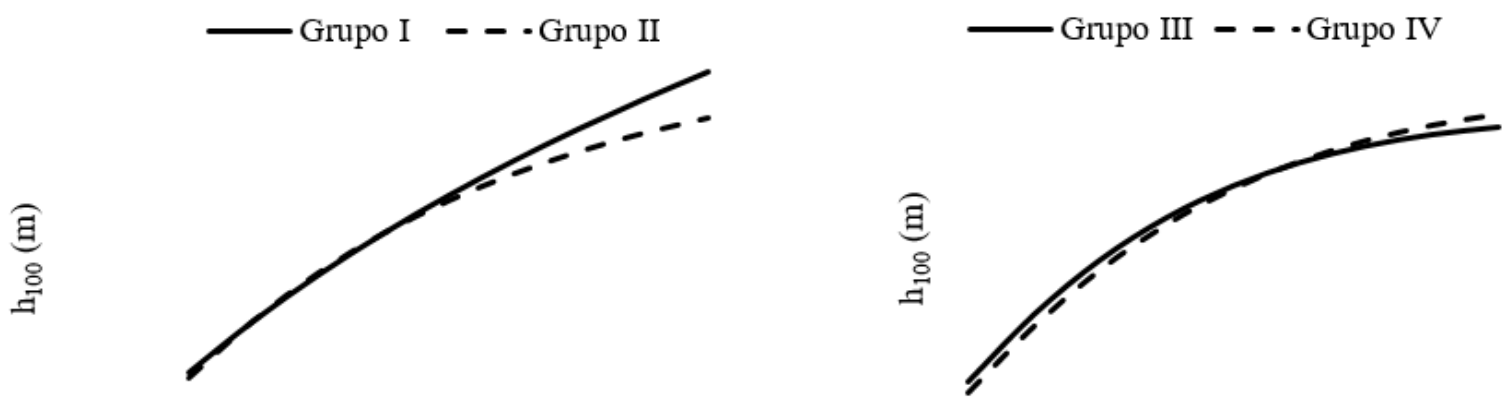

Idade (anos)

Idade (anos)

FIGURA 3: Curvas ajustadas pelo modelo de Backman para os grupos I, II, III e IV, após análise do fator solo.

FIGURE 3: Fitted curves by Backman's model for the groups I, II, III and IV, after the soil factor analysis. 
TABELA 7: Significância representada pelo valor de probabilidade "p" entre as classes de aptidão de solos agrupadas pela análise de covariância para o clone 2864 e clone 4039.

TABLE 7: Significance demonstrated by the probability "p" value among different grouped soil classes, by using the analysis of covariance for clones 2864 and 4039.

\begin{tabular}{ccccc}
\hline & Classe/Classe & $1-2$ & 3 & $4-5$ \\
Clone 2864 & $1-2$ & -- & 0.00000 & 0.00348 \\
& 3 & 0.00000 & -- & 0.03809 \\
& $4-5$ & 0.00348 & 0.03809 & -- \\
\hline \multirow{4}{*}{ Clone 4039} & Classe/Classe & $1-2$ & 3 & $4-5$ \\
& $1-2$ & -- & 0.61504 & 0.00034 \\
& 3 & 0.61504 & -- & 0.00041 \\
\hline
\end{tabular}

Em que: Valores de probabilidade $\mathrm{p}<0,01$ em negrito indicam diferenças significativas de inclinação ou nível pela análise de covariância entre as diferentes classes de aptidão de solos sob um nível de probabilidade de erro de $1 \%$.

\section{CONCLUSÕES}

Este estudo permitiu concluir que existem diferentes tendências de crescimento em altura dominante para os dois materiais genéticos avaliados e para as distintas classes de aptidão de solos. Para cada grupo definido deverá se proceder separadamente o ajuste e classificação de sítio, de forma que as demais relações funcionais, normalmente presentes nos modelos de crescimento e produção, sejam embasadas nestas funções individualizadas para cada sítio.

\section{AGRADECIMENTOS}

Meu especial agradecimento à CMPC Celulose Riograndense Ltda. pelo apoio e disponibilização de informações para a realização deste trabalho.

\section{REFERÊNCIAS}

BARROS, N. F. et al. Análise do crescimento de Eucalyptus saligna em solo de cerrado sob diferentes níveis de N. P. e K. no Vale do Jequitinhonha, MG. IPEF, Piracicaba, n. 26, p. 13-17, abr. 1984.

BELlOTE, A. F. J.; DEDECEK, R. A. Atributos Físicos e Químicos do Solo e suas Relações com o Crescimento e a produtividade do Pinus taeda. Boletim Pesquisa Florestal, Colombo, n. 53, p. 21-38, jul./dez. 2006.

CAMPOS, J. C. C.; LEITE, H. G. Mensuração florestal: perguntas e respostas. 3. ed. Viçosa: Ed. UFV, 2009. $548 \mathrm{p}$.

CARVALHO, A. P. et al. Efeitos de características do solo sobre a capacidade produtiva de Pinus taeda.

Boletim de Pesquisa Florestal, Colombo, n. 39, p. 51-66, jul./dez. 1999.

CLUTTER, J. L.; FORTSON, J. C.; PIENAAR, L. V. Timber Management: a quantitative approach. New York: John Wiley \& Sons, 1983. 329 p.

COSTA, A. M. et al. Unidades de manejo para o cultivo de eucalipto em quatro regiões fisiográficas do Rio Grande do Sul. Scientia Forestalis, Piracicaba, v. 37, n. 84, p. 465-473, dez. 2009.

FINGER, C. A. G. et al. Influência da camada de impedimento no solo sobre o crescimento de Eucalyptus grandis (hill) ex Maiden. Ciência Florestal, Santa Maria, v. 6, n. 1, p. 137-145, 1996.

FREITAS, D. A. F. et al. Índices de qualidade do solo sob diferentes sistemas de uso e manejo florestal e cerrado nativo adjacente. Revista Ciência Agronômica, Ceará, v. 43, n. 3, p. 417-428, jul./set. 2012.

GONÇALVES, J. L. M. Interpretação de levantamentos de solos para fins Silviculturais. IPEF, Piracicaba, 
n. 39, p. 65-72, ago. 1988.

KOPPEN, W. Grundriss der Klimakunde. Berlin: Walter de Gruyter, 1931. 390 p.

LIBARDI, P. L. Água no solo. In: LIER, Quirijn de Jong van (Ed). Física do Solo. 1. ed. Viçosa, MG: Sociedade Brasileira de Ciência do Solo, 2010. p. 103-152.

LOURENÇO, M. F. S. Características limitantes do solo no crescimento de Eucalyptus dunnii Maiden, em uma toposeqüiência na região de três barras (SC). 1996. 140 f. Dissertação (Mestrado em Engenharia Florestal) - Universidade Federal do Paraná, Curitiba, 1996.

MELO FILHO, J. F.; SOUZA, A. L. V.; SOUZA, L. S.; Determinação do índice de qualidade subsuperficial em um latossolo amarelo coeso dos tabuleiros costeiros, sob floresta natural. Revista Brasileira de Ciência do Solo, n. 31, p. 1599-1608, 2007.

MENDES, I. C. et al. Cálculo de um índice de qualidade do solo para diferentes agroecossistemas do cerrado. In: SIMPÓSIO NACIONAL DO CERRADO, 9., 2008, Brasília, DF. Anais... 2008. p. 12-17.

MORENO, J. A. Clima do Rio Grande do Sul. Porto Alegre: Secretaria da Agricultura, 1961. 41 p.

ORTIZ, J. L. et al. Relações espaciais entre o potencial produtivo de um povoamento de eucalipto e atributos do solo e do relevo. Scientia Forestalis, Piracicaba, n. 72, p. 67-79, dez. 2006.

SCOLFORO, J. R. Curvas de índice de sítio para Pinus caribaea var.hondurensis. IPEF, Piracicaba, n. 45, p. 40-47, jan./dez. 1992.

SELLE, G. L. Guias de densidade e índices de sítio para Hovenia dulcis Thunberg na região central do estado do Rio Grande do Sul, Brasil. 2009. 97 f. Tese (Doutorado em Engenharia Florestal) - Universidade Federal de Santa Maria, Santa Maria, 2009.

SILVA, J. A. A.; BAILEY, R. L. Uso de transformações normalizadoras no ajuste de modelos volumétricos. Revista Árvore, Viçosa, MG, v. 15, n. 12, p. 199-206, 1991.

STRECK, E. V. et al. Solos do Rio Grande do Sul. 2. ed. Porto Alegre: EMATER/RS; UFRGS, 2008. $222 \mathrm{p}$.

TONINI, H. Crescimento e produção de clones de Eucalyptus saligna Smith, na depressão central e serra do sudeste, Rio Grande do Sul. 2003. 331 f. Tese (Doutorado em Engenharia Florestal) Universidade Federal de Santa Maria, Santa Maria, 2003.

TONINI, H. et al. Índice de sítio para Pinus elliottii Engelm, em três unidades de mapeamento de solo, nas regiões da serra do sudeste e litoral, no estado do Rio Grande do Sul, BRASIL. Ciência Florestal, Santa Maria, v. 12, n. 2, p. 61-73. 2002.

TONINI, H.; SCHNEIDER, P. R.; FINGER, C. A. G. Curvas de índice de sítio para povoamentos clonais de Eucalyptus saligna Smith para a depressão central e serra do sudeste, Rio Grande do Sul. Ciência Florestal, Santa Maria, v. 16, n. 1, p. 27-43, 2006.

TRUGILHO, P. F. et al. Avaliação de Clones de Eucalyptus para produção de carvão vegetal. Cerne, Lavras, v. 7, n. 2, p. 104-114, 2001. 